\title{
Dynamics of population of gadolinium-156 nuclei energy levels during neutron pumping of isotope-modified gadolinium oxide
}

\author{
Igor V. Shamanin ${ }^{\mathrm{a}}$, Mishik A. Kazaryan ${ }^{\mathrm{b}}$, Sergei V. Bedenko ${ }^{\mathrm{a}}$, Vladimir.V. Knysheva, \\ Vitaly I. Shamanin ${ }^{\mathrm{a}}$ \\ ${ }^{a}$ National research Tomsk polytechnic university, prospekt Lenina, 30, 634050, Tomsk, Russia; \\ ${ }^{\mathrm{b}}$ Physical institute RAS, Leninsky prospekt, 53, 119991, Moscow, Russia
}

\begin{abstract}
The possibility of transformation of energy of fast and epithermal neutrons to energy of coherent photon radiation at the expense of a neutron pumping of the active medium formed by nucleus with long-living isomerous states is theoretically described. The channel of the nucleus formation in isomeric state as a daughter nucleus resulting from the nuclear reaction of neutron capture by a lighter nucleus is taken into consideration for the first time. The analysis of cross sections dependence of radiative neutron capture by the nuclei of gadolinium isotopes $\mathrm{Gd}^{155}$ and $\mathrm{Gd}^{156}$ is performed. As a result, it is stated that the speed of $\mathrm{Gd}^{156}$ nuclei formation exceeds the speed of their "burnup" in the neutron flux. It is provided by a unique combination of absorbing properties of two isotopes of gadolinium $\mathrm{Gd}^{155}$ and $\mathrm{Gd}^{156}$ in both thermal and resonance regions of neutron energy. The possibility of excess energy accumulation in the participating medium created by the nuclei of the pair of gadolinium isotopes $\mathrm{Gd}^{155}$ and $\mathrm{Gd}^{156}$ due to formation and storage of nuclei in isomeric state at radiative neutron capture by the nuclei of the stable isotope with a smaller mass is shown. It is concluded that when the active medium created by gadolinium nuclei is pumped by neutrons with the flux density of the order of $10^{13} \mathrm{~cm}^{-2} \cdot \mathrm{s}^{-1}$, the condition of levels population inversion can be achieved in a few tens of seconds. The wave length of the radiation generated by the medium is $0.0006 \mathrm{~nm}$.
\end{abstract}

Keywords: gadolinium isotopes, active medium, neutron pumping, inversion of energy levels population.

\section{INTRODUCTION}

Active medium is considered to be some matter in which it is possible to create the nucleus energy level population inversion due to radiation capture reaction and inelastic neutron scattering by the nuclei present in the matter.

The combination of nuclear transformations occurring in the matter under the influence of the neutron flux is called nuclide kinetics. Differential and integral characteristics of nuclide kinetics determine isotopic composition of the matter which was or is in the neutron field. At present the nuclide kinetics investigation results are applied mainly in physics and nuclear reactors engineering ${ }^{[1]}$ and in particular their nuclear safety. The possibility of accumulation and uncontrolled release of excess energy in neutron-absorbing materials because of potential accumulation of excess energy in isomeric states of atomic nuclei (for example, hafnium or gadolinium) comprising some of them was paid attention to ${ }^{[2]}$.

\subsection{Theoretical evaluation}

Let us consider the neutron-absorbing material in which the following processes occur under the influence of neutrons: nucleus $\mathrm{X}+$ neutron $\rightarrow$ nucleus $\mathrm{Y}$ in the excited state $\rightarrow$ nucleus $\mathrm{Y}$ in the isomeric (metastable) state $\rightarrow$ nucleus $\mathrm{Z}$ in the ground state. For example: $\mathrm{Gd}^{155}+\mathrm{n} \rightarrow \mathrm{Gd}^{156^{*}} \rightarrow \mathrm{Gd}^{156 \mathrm{~m}} \rightarrow \mathrm{Gd}^{156}$. Nuclei $\mathrm{Y}$ and $\mathrm{Z}$ also undergo radiation capture, that is they are "shot". Isomer $\mathrm{Gd}^{156 \mathrm{~m}}$ has the half-life period of $1.3 \mu$ s and decays emitting gamma-quantum with the energy of $2.1376 \mathrm{MeV}$. In figure 1 the scheme of the process is shown.

* shiva@tpu.ru; phone +7 3822 606112; fax +7 3822 423934; tpu.ru

**kazar@ sci.lebedev.ru; phone +7 499132 6432; lebedev.ru

XIV International Conference on Pulsed Lasers and Laser Applications, edited by Victor F. Tarasenko, Anton V. Klimkin, Maxim V. Trigub, Proc. of SPIE Vol. 11322, 1132212 · @ 2019 SPIE CCC code: $0277-786 \mathrm{X} / 19 / \$ 21 \cdot$ doi: $10.1117 / 12.2550338$ 


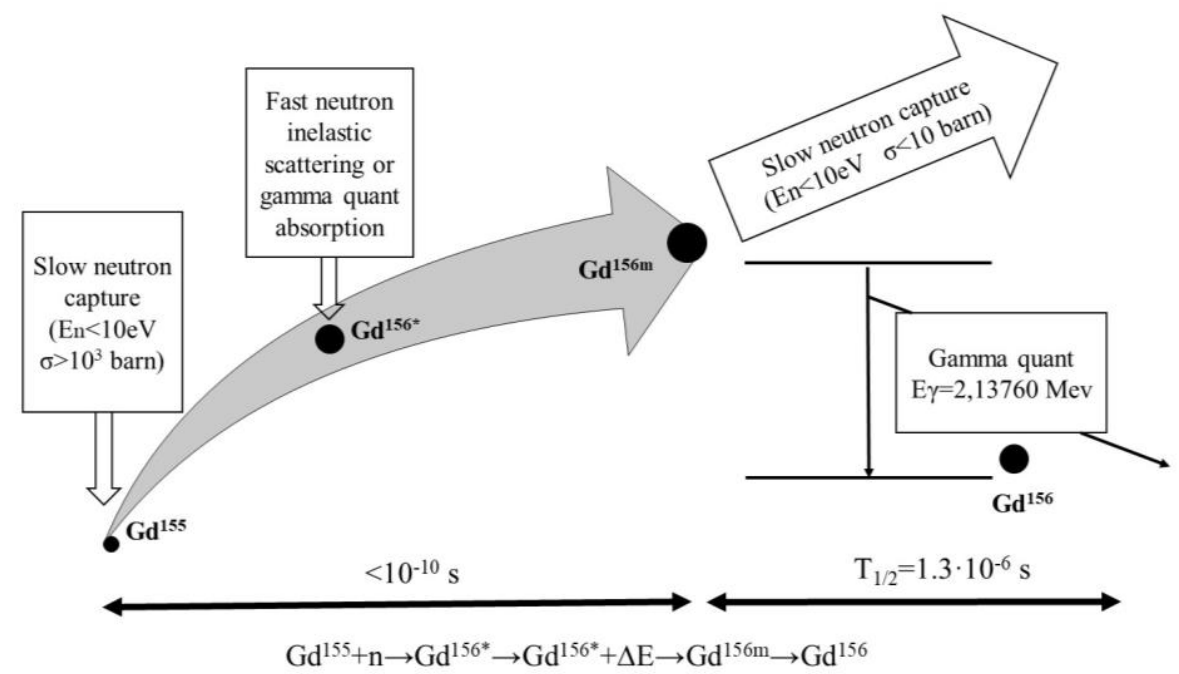

Figure. 1 Pumping of the active medium formed by gadolinium isotopes nuclei ${ }^{[3]}$.

In table 1 the parameters of two Gd isotopes in ground and isomeric states are presented.

Table. 1. Parameters of gadolinium isotopes nuclei.

\begin{tabular}{|c|c|c|c|}
\hline Nucleus & Half-life & $\begin{array}{c}\text { Isotopic content in } \\
\text { natural mixture }\end{array}$ & Spin and nucleus parity \\
\hline $\mathbf{G d}^{\mathbf{1 5 5}}$ & Stable & $14.80 \%$ & $3 / 2-$ \\
\hline $\mathbf{G d}^{\mathbf{1 5 5 m}}$ & $31.97 \mathrm{~ms}$ & - & $11 / 2-$ \\
\hline $\mathbf{G d}^{\mathbf{1 5 6}}$ & Stable & $20.47 \%$ & $0+$ \\
\hline $\mathbf{G d}^{\mathbf{1 5 6 m}}$ & $1.3 \mu \mathrm{s}$ & - & $7-$ \\
\hline
\end{tabular}

Before appearing in a metastable state, $\mathrm{Gd}^{156}$ nucleus is in an excited state. The typical nucleus lifetime in the excited state is $\sim 10-14 \mathrm{~s}$, which is nine orders of magnitude longer than the nuclear interaction time. Therefore, the nucleus in the excited state can gain and conserve energy $\Delta \mathrm{E}$ transferred to it as a result of neutron scattering on it. Energy supplied to the nucleus when the neutron dissipates on it depends on the nucleus mass - the less the nucleus mass is, the bigger energy the neutron gives to it during dissipation. In the ideal case, energy $\Delta E$ transferred at dissipation is the value equal to the difference between the energy of metastable state and energy of excitation. For the given value of the neutron energy $E_{\mathrm{n}}$ the value of the energy $\Delta E$ transferred at inelastic scattering on the nucleus can be determined from the transcendent equation:

$$
\frac{\Delta E^{i}}{E_{n}}=\frac{2 A}{(A+1)^{2}}\left(1+\frac{A+1}{2} \cdot \frac{\Delta E^{i}}{E_{n}}-0.07 A^{2 / 3} E_{n} \sqrt{\left.1-\frac{A+1}{A} \cdot \frac{\Delta E^{i}}{E_{n}}\right),}\right.
$$

where $\mathrm{A}$ - the nucleus mass number. The equation is obtained on the assumption that the transferred energy $\Delta E$ is equal to the energy of nucleus excitation from the ground state. If the nucleus has several excitation levels $(i=1,2,3 \ldots)$, the equation allows determining the value of the neutron energy $E_{n}$, providing transfer of the nucleus to the corresponding excitation level.

The number of collisions (dissipations) of the neutrons with the nuclei of active medium occurring in the medium volume unit per time unit can be determined by the following relation:

$$
\Phi \sigma n_{n u c},
$$

where $\Phi$ - the neutron flux density, $\sigma$ - microscopic cross section of inelastic neutron scattering on the nuclei, $\mathrm{n}_{\text {nuc }}-$ the number of nuclei per medium volume unit. In this case the scattering frequency experienced by neutrons in the active medium is determined by the following relation: 
where $v$ - neutron velocity $(v=\sqrt{2 E / m})$

For example, to transfer nuclei of ${ }_{54} \mathrm{Xe}^{130}$ isotope from the ground state to the excited state, taking ino account that they have 3 excitation levels $(0.54 ; 1.21$ and $1.95 \mathrm{MeV})$, the presence of neutrons with the energies of $0.709 ; 1.285$ and 2.005 $\mathrm{MeV}$, correspondingly, is required in the flux. To transfer nuclei of ${ }_{10} \mathrm{Ne}^{22}$ isotope from the ground state to the excited state, which also has 3 excitation levels, the presence of neutrons with the energies of $2.075 ; 3.747$ and $4.859 \mathrm{MeV}$ is required in the flux. The average neutron energy of the fission spectrum is $2 \mathrm{MeV}$. The average neutron spectrum energy of the nuclear reactor (even fast neutron reactors) is significantly lower. Besides, to transfer isotope nuclei to the excited state by direct scattering of neutrons on nuclei it is necessary to "choose" isotopes not only with bigger specific binding energy of nucleons in the nucleus, but also with small value of the neutron absorption cross section. Therefore, to accumulate nuclei in the excited state, it is reasonable to obtain them as a product of the reaction of neutron radiative capture by nuclei with a mass number smaller by one unity. The daughter nucleus is formed in the excited state and, if required, gains an additional energy due to neutron scattering on it. As a result, the daughter nucleus appears in the metastable state.

To evaluate the possibility of energy accumulation in isomeric nuclei states due to radiation neutron capture in such material it is required to solve the differential equations system ${ }^{[3]}$ :

$$
\left\{\begin{array}{l}
\frac{d x}{d t}=-\sigma_{1} x \Phi \\
\frac{d y}{d t}=\sigma_{1} x \Phi-\sigma_{2} y \Phi-\lambda y \\
\frac{d z}{d t}=-\sigma_{3} z \Phi+\lambda y
\end{array}\right.
$$

Here $x(t), y(t), z(t)-\mathrm{Gd}^{155}, \mathrm{Gd}^{156 \mathrm{~m}}, \mathrm{Gd}^{156}$ nuclei concentration, respectively; $\Phi$ - neutron flux density; $\sigma$ - micro cross section of radiation neutron capture $\left(\sigma_{1}\right.$ - for $\mathrm{Gd}^{155}$ nuclei, $\sigma_{2}$ - for $\mathrm{Gd}^{156 \mathrm{~m}}$ nuclei, $\sigma_{3}$ - for $\mathrm{Gd}^{156}$ nuclei), $\lambda$ - the decay constant of isomers nuclei $\mathrm{Gd}^{156 m}$.

When neutrons with the flux density $\Phi=10^{13} \mathrm{~cm}^{-2} \cdot \mathrm{s}^{-1}$ influence the neutrons absorber formed by gadolinium nuclei, the condition $\frac{y(t)}{z(t)} \approx 1$ is achieved within several tens of seconds. It is explained by almost unique combination of absorbing properties of two isotopes of gadolinium $\left(\mathrm{Gd}^{155}\right.$ and $\left.\mathrm{Gd}^{156}\right)$ in both thermal and resonant energy regions of neutrons.

An analysis of the dependences of the micro-cross sections of neutron radiative capture by gadolinium isotopes on the neutron energy, presented in paper ${ }^{[4]}$, indicates a favourable combination of properties of two gadolinium isotopes with mass numbers of 155 and 156, consisting in preferential absorption of neutrons by the light isotope in a rather wide energy range.

The reaction cross section of the neutron radiative capture by $\mathrm{Gd}^{155}$ nuclei exceeds by 3-4 orders of magnitude that for $\mathrm{Gd}^{156}$ nuclei at neutron energies to $10 \mathrm{eV}$; the resonance integral for $\mathrm{Gd}^{155}$ nuclei significantly exceeds that for $\mathrm{Gd}^{156}$ nuclei. The production rate of $\mathrm{Gd}^{156 \mathrm{~m}}$ nuclei is significantly higher than their "shooting" by neutrons and the rate of their ground-state transition even at flux densities of resonant and thermal neutrons of the order of $\sim 10^{13} \mathrm{~cm}^{-2} \cdot \mathrm{s}^{-1}$. The further increase in the neutron flux density leads to reducing the time interval after which the excess energy begins to accumulate. As a result, rapid accumulation of excess energy in the metastable state of gadolinium-156 isotope nuclei should be expected at moderate neutron flux densities. The possibility of pumping the medium formed by gadolinium nuclei with gamma-quanta was studied in paper ${ }^{[5]}$. External gamma-quantum flow cannot provide conditions for population inversion of metastable nuclei energy levels. 


\section{CALCULATION RESULTS}

To perform the research of excess energy accumulation in $\mathrm{Gd}$ the following system to place $\mathrm{Gd}_{2} \mathrm{O}_{3}$ in the reactor core was used (see Figure. 2).

$\mathrm{Gd}_{2} \mathrm{O}_{3}$ is placed in a cylindrical volume made of pure tungsten. Further the cylinder is placed in the active core of the reactor unit. Uranium-graphite reactor is chosen as a reactor unit for the purpose of investigating the sample in thermal neutrons spectrum ${ }^{[6-8]}$.

Several versions of the tungsten bulb with graphite reflector and without reflector are considered in the work (see Table 2). Graphite block serves as a reflector. Isotopic composition of $\mathrm{Gd}$ consists of $50 \% \mathrm{Gd}^{155}$ and $50 \% \mathrm{Gd}^{156}$.

Table. 2 - Geometrical characteristics of the tungsten bulb with $\mathrm{Gd}_{2} \mathrm{O}_{3}$

\begin{tabular}{|c|c|c|c|c|c|c|}
\hline & \multicolumn{7}{|c|}{$\mathbf{d 1}, \mathbf{c m}$} & $\mathbf{d 2}, \mathbf{c m}$ & $\mathbf{d 3}, \mathbf{c c m}$ & $\mathbf{h 1}, \mathbf{c m}$ & h2, cm \\
\hline & \multicolumn{7}{|c|}{ Without reflector } \\
\hline 5_get & 5 & 7 & - & 30 & 31 \\
\hline 10_get & 10 & 12 & - & 30 & 31 \\
\hline & & \multicolumn{7}{|c|}{ With the reflector } \\
\hline 5_get+C & 5 & 7 & 30 & 30 & 31 \\
\hline 10_get+C & 10 & 12 & 30 & 30 & 31 \\
\hline
\end{tabular}

The neutronic calculation was performed using a WIMSD-5B.12 specialized program (OECD Nuclear Energy Agency). The program WIMS is applied for calculation of thermal and fast reactors. It is also successfully used for designing reactors and calculation and analysis of various effects in current reactor units. At present the program uses the universal 69-group library of constants made on the basis of the evaluated neutron data files (ENDF, JEF, JENDL и т.д.) ${ }^{[8-11]}$.

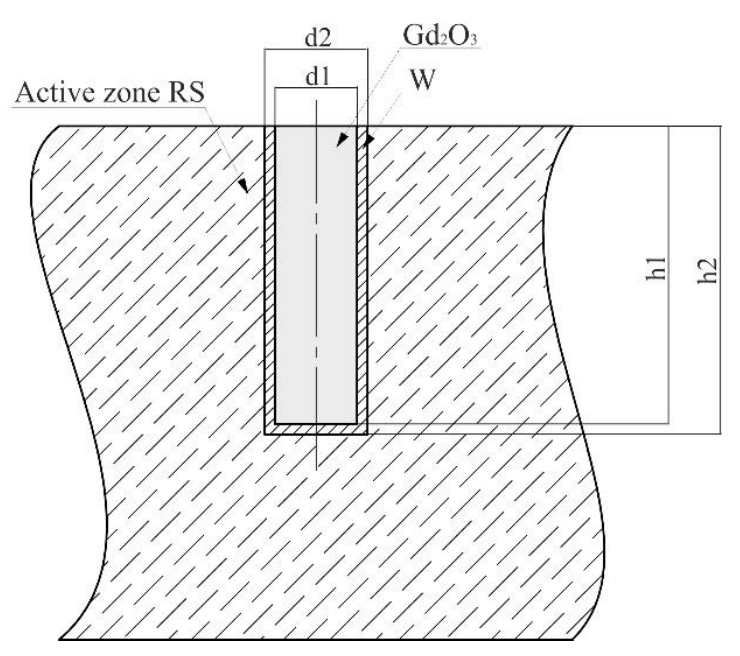

a)

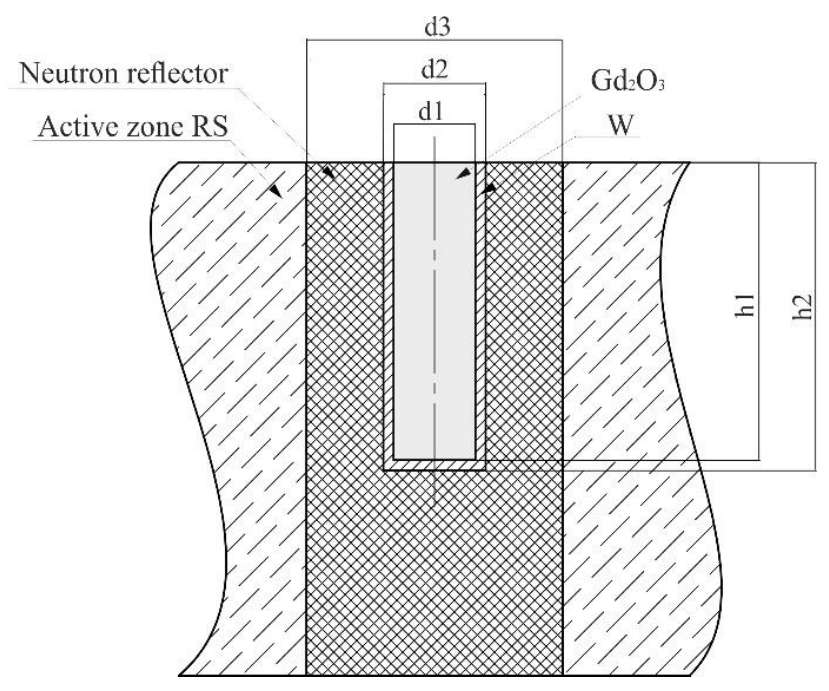

b)

Figure. 2 - The scheme of tungsten bulb placement in the reactor unit: a) without the reflector; b) with the reflector.

The calculation was performed for all variants of the placed sample. To compare and analyze the obtained results a preliminary calculation of the initial spectrum in the placement region of the sample in the reactor active core. The spectrum calculation results in the placed sample are presented in Figure. 3, 4. Changes in the reactor's neutron spectrum at the location of a cylinder made of $\mathrm{Gd}_{2} \mathrm{O}_{3}$ suggest that the rate of "production" of metastable $\mathrm{Gd}^{156 \mathrm{~m}}$ nuclei will 
significantly exceed their "burnout" rate in the neutron field. Simultaneous fulfillment of the condition $\frac{y(t)}{z(t)}>1$ will lead to the generation in the $\mathrm{Gd}_{2} \mathrm{O}_{3}$ volume of radiation with a wavelength of $0.0006 \mathrm{~nm}$.

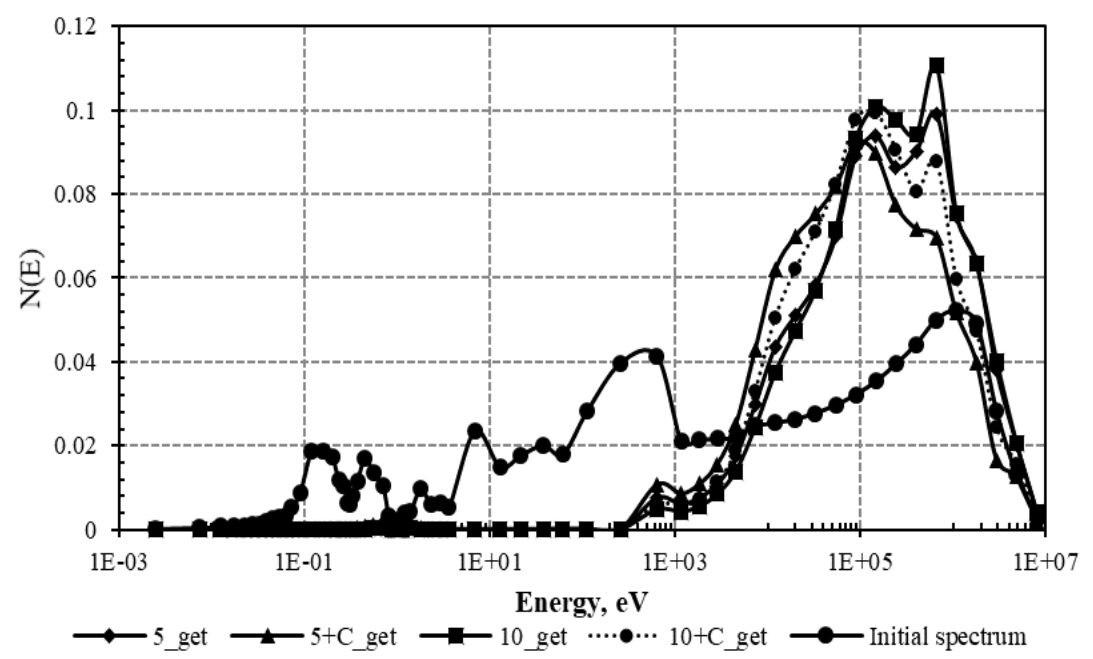

Figure. 3 - Total neutron spectrum

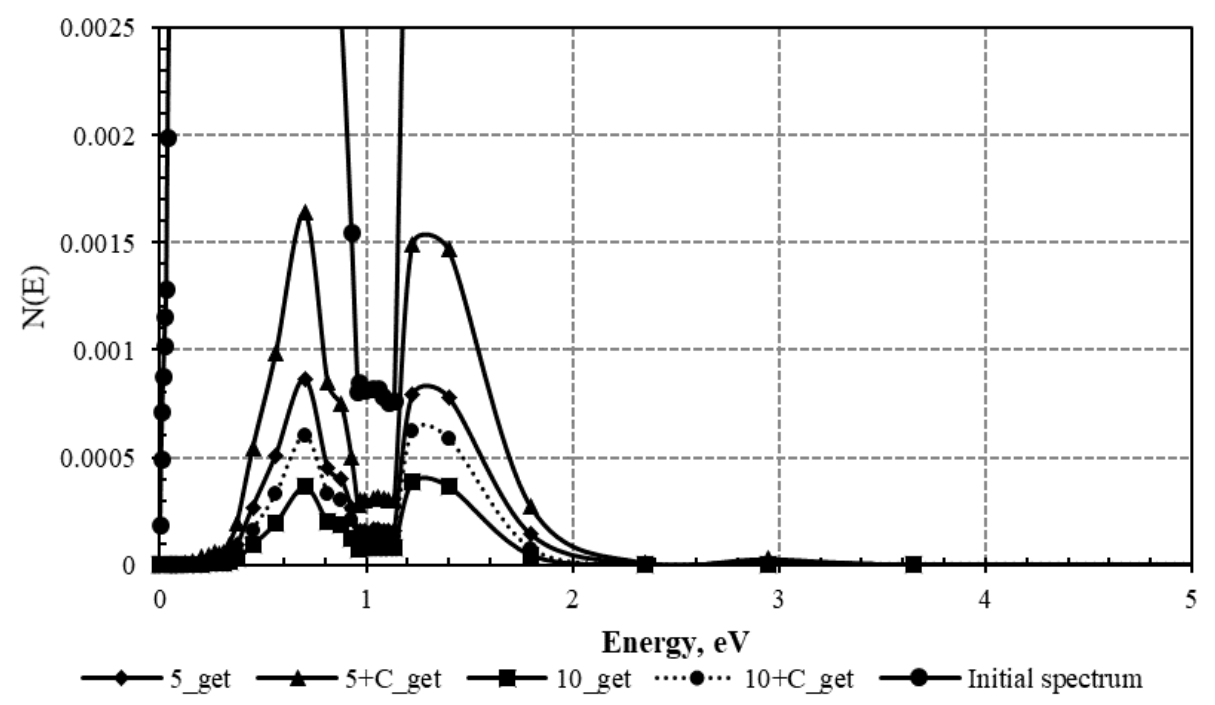

Figure. 4 - the spectrum in the energy range from 0 to $5 \mathrm{eV}$

\section{CONCLUSION}

Accumulation of excess energy in active medium formed by the nuclei of stable isotopes of gadolinium with mass numbers of 155 and 156 due to formation of atomic nuclei in isomeric state at radiation capture of neutrons by the nuclei with the smaller mass is possible. Pumping the active medium created by gadolinium nuclei by the neutrons with the flux density $\Phi$ equal to $10^{13} \mathrm{~cm}^{-2} \cdot \mathrm{s}^{-1}$ the condition of the population inversion can be achieved within several tens if seconds. The wavelength of the radiation generated by the medium is $0.0006 \mathrm{~nm}$. Sintered ceramics on the basis of $\mathrm{Gd}_{2} \mathrm{O}_{3}$ enriched by the $155^{\text {th }}$ isotope can be considered as possible active medium. The active medium is placed in a cylindrical volume made of tungsten, which is characterized by a relatively small (to 1 barn) neutron capture cross section in a wide neutron energy range. 


\section{REFERENCES}

[1] Shamanin, I. V., Bedenko, S. V., Pavlyuk, A. O., Lyzko, V. A. "Using the ORIGEN-ARP program in calculating the isotopic composition of spent fuel from the VVER-1000 reactor", Bulletin of the Tomsk polytechnic university, V. 317(4), 25-28 (2010).

[2] Shamanin, I. V., Kazaryan, M. A., "Nuclide kinetics involving hafnium and gadolinium nuclei in long-lived isomeric states", Bulletin of the Lebedev Physics Institute, V. 44(7), 215-217 (2017).

[3] Kazaryan, M. A., Shamanin, I. V., Shamanin, V. I., Sachkov, V. I., Poberezhnikov, A. D. "A mechanism for creating an inversion of populations of energy levels", "International Conference on Atomic and Molecular Pulsed Lasers XIII; 1061416, Tomsk, Russia (2018)

[4] Evaluated Nuclear Data File (ENDF) https://www-nds.iaea.org/exfor/endf.htm. Date of the application 27.04.2019.

[5] Tkalya, Ye. V. "Induced decay of a nuclear isomer $\mathrm{Hf}^{178 \mathrm{~m} 2}$ and an "isomeric bomb"”, Advances in Physical Sciences, V. 175(5), 555-561 (2005).

[6] Lyovina, I. K., Sidorenko, V. A. "Some neutron-physics considerations of improving the fuel use in watercooled thermal VVÉR and RBMK power reactors", Soviet Atomic Energy, V. 60(4), 283-287 (1986).

[7] Kulikov, E. V. "State-of-the art and development prospects for nuclear power stations containing RBMK reactors", Soviet Atomic Energy, V. 56(6), 368-374 (1984).

[8] Romanenko, V. S., Krayushkin, A. V. "Physical characteristics of an RBMK reactor in the transitional period", Soviet Atomic Energy, V. 53(6), 816-824 (1982).

[9] Lindley, B. A., Hosking, J. G., Smith, P. J., Powney, D. J., Tollit, B. S., Newton, T. D., Perry, R., Ware, T. C., Smith, P. N. "Current status of the reactor physics code WIMS and recent developments", Annals of Nuclear Energy, V. 102, 148-157 (2017).

[10] Shamanin, I. V., Grachev, V. M., Chertkov, Y. B., Bedenko, S. V., Mendoza, O., Knyshev, V. V. "Neutronic properties of high-temperature gas-cooled reactors with thorium fuel, Annals of Nuclear Energy, V. 113, 286293 (2018).

[11] Galchenko, V., Mishyn, A. "Comparative analysis of reactor cycle neutron characteristics using different WIMSD5B nuclear data libraries", Nuclear and Radiation Safety, V. 3(67), 8-12 (2015). 